\title{
OPTIMIZATION AND RESEARCH OF HIGH-VOLTAGE VOLTAGE CONVERTER CIRCUITS FOR PLANT SPRAYING SYSTEMS WITH ELECTROAEROSOLS
}

\author{
Sergey Antonov, Andrey Adoshev, Ildar Sharipov, Alexander Ivashina \\ Stavropol State Agrarian University, Russia \\ antonov_serg@mail.ru, adoshev@mail.ru,sh_ik@mail.ru,av_ivashina49@mail.ru
}

\begin{abstract}
The methods of the electroaerosol technology are based on the use of electrostatic field forces and the properties of electric discharge in gases in technological processes. In agriculture, the use of electroaerosols has been used to protect plants. High voltage devices are used to create an electric field. When designing these devices, problems arise with high voltage sources. This is due primarily to the insulating properties of the circuit elements. It is necessary to carry out breakdown voltage calculations on the case and external elements of the device. For plant spraying systems, push-pull half-bridge high-voltage sources are most optimal. The main task in optimizing a high voltage source is to select a circuit, to determine the modes and characteristics of voltage. Designing a power source includes: determining operating modes and selecting power transistors; calculation and selection of elements of the voltage multiplier; determination of design parameters of a power transformer. The multiplier consists of elementary links. When combining several elementary links, at each subsequent node in the diode circuit, the constant component of the potential increases by the amplitude of the source. The proposed principles make it possible to obtain an algorithm for constructing a multiplier of any degree of complexity. Structurally, the most technologically advanced option is the use of components for surface mounting. To determine the parameters of the multiplier, numerical analysis (modeling) of a specific device and optimization of the circuit parameters to obtain the required characteristics are used. Among the advantages of the method can be called simplicity, quick results, sufficiently high accuracy, the ability to take into account the features of the elements used when using complex models for them. Experimentally output characteristics of the power source and voltage multiplier at real load are obtained.
\end{abstract}

Keywords: high voltage, transformer, voltage multiplier, electric aerosol, power transistor.

\section{Introduction}

For successful development of agriculture, it is necessary to pay attention to increasing agricultural production. The main problem of increasing the production volume is the reduction of land fertility and losses associated with pests, diseases and weediness of fields. The use of mineral, organic fertilizers, plant protection products is one of the factors of agricultural production intensification [1-3]. Using electric charging of aerosols makes it possible to increase the efficiency of foliar application and plant protection products [4-6].

One of the effective methods of spraying plants is presented in the article [7]. It consists in air ionization and its further mixing with fertilizer solutions or plant protection products. The main task in this case is the development of a high voltage source. The main element of a high voltage source is a voltage multiplier. Therefore, at the beginning, it is necessary to consider the device and the operating principle of the voltage multiplier in a general form. Based on this, it is needed to study not one specific type of multiplier, but the entire class of possible multipliers. Such an approach will allow us to obtain an algorithm for constructing a multiplier of any degree of complexity and find a universal explanation of the multiplier operating principle, suitable for a device with any structure. To achieve these goals, it is necessary to consider the following questions: the generalized structure of the multiplier, the multiplier operating principle, the construction of the multiplier with an arbitrary multiplication factor, the search for all possible multipliers, the parameters of this multiplier type.

In the article [8] it was said that the initial data for the design of a high voltage source are: technical, operational and economic parameters. Many of the requirements are the opposite. Therefore, it is necessary to determine particularly important parameters, on the basis of which compromise decisions are made.

A model scheme of the voltage multiplier is shown in Fig. 1.

At the initial stage of designing the voltage multiplier, it is necessary to describe the operating principle of the device, give an algorithm for constructing the multiplier from elementary links, study the transient process when turned on, analyze the operation in the steady state under load, derive formulas for calculating the multiplier with an arbitrary multiplication factor. 


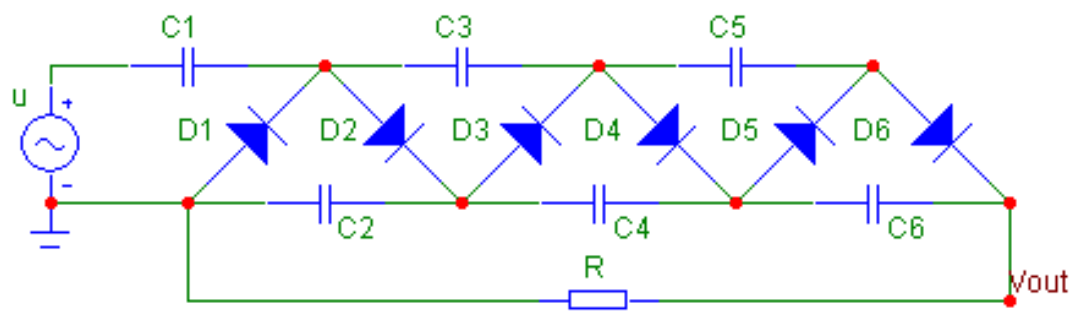

Fig. 1. Voltage multiplier structure

\section{Materials and methods}

It must be understood that a circuit of series-connected diodes does not have the properties of voltage multiplication. To get a multiplier, the circuit must be supplemented with capacitors connected in a certain way.

First, we need to consider the operation of the load-free circuit, in which there is an AC voltage source with amplitude $U_{a}$, and point A with potential $n \cdot U_{a}$ (for example, the output of the multiplier that we want to increase to obtain the coefficient $n+2$; in particular, it can be a point with potential 0 - common wire or $U_{a}$ - output of a half-wave rectifier).

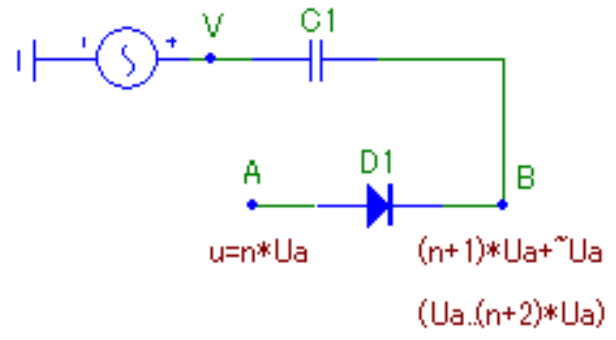

Fig. 2. Connecting elements to a power source

We connect between point $\mathrm{A}\left(u=n \cdot U_{a}\right)$ and $\mathrm{AC}$ voltage source a circuit from the diode $\mathrm{D} 1$ and capacitor C1 (Fig. 2.). The blocking capacitor prevents the closing of point A to the power source when the diode is on-state. At the moment when the instantaneous voltage of the source reaches the value $u=-U_{a}$, the potential difference between points $\mathrm{A}$ and $\mathrm{V}$ reaches the value

$$
n \cdot U_{a}-\left(-U_{a}\right)=n \cdot U_{a}+U_{a}=(n+1) \cdot U_{a},
$$

which means that in the steady state, the capacitor $\mathrm{C} 1$ will be charged to voltage $(n+1) \cdot U_{a}$ and due to the presence of the diode $\mathrm{D} 1$, the voltage on it will not change. Therefore, at point $\mathrm{B}$, the voltage equal to the sum of the instantaneous voltage of the source and the voltage across the capacitor C1 will contain a constant component $(n+1) \cdot U_{a}$ and a variable component with amplitude $U_{a}$. In this case, the potential of point B will periodically change between the minimum value $n \cdot U_{a}$ and maximum value $(n+2) \cdot U_{a}$.

The structural diagram of an inverter high-voltage source should contain: auxiliary source, inverter, transformer, control circuit, voltage multiplier, ionizer [9].

The inverter converts the DC voltage of the on-board power system into AC voltage of high frequency, which is fed to the transformer, where the conversion takes place according to the given control laws. The conversion frequency reaches $50-100 \mathrm{kHz}$, this can significantly reduce the overall dimensions of the transformer and the converter as a whole.

The switching order of the inverter power transistors is set by a control circuit that stabilizes the output voltage of the converter at a level specified by the reference voltage at the input of the circuit $U_{\text {in }}$ due to feedback on the output voltage $U_{\text {out }}$ by changing the duty cycle of the power transistor control pulses. Additionally, the control circuit generates fixed dead time pauses between switching power transistors to exclude the possibility of through-currents of the inverter, while delaying the process of turning off the transistors due to variable thermal loads [9]. 


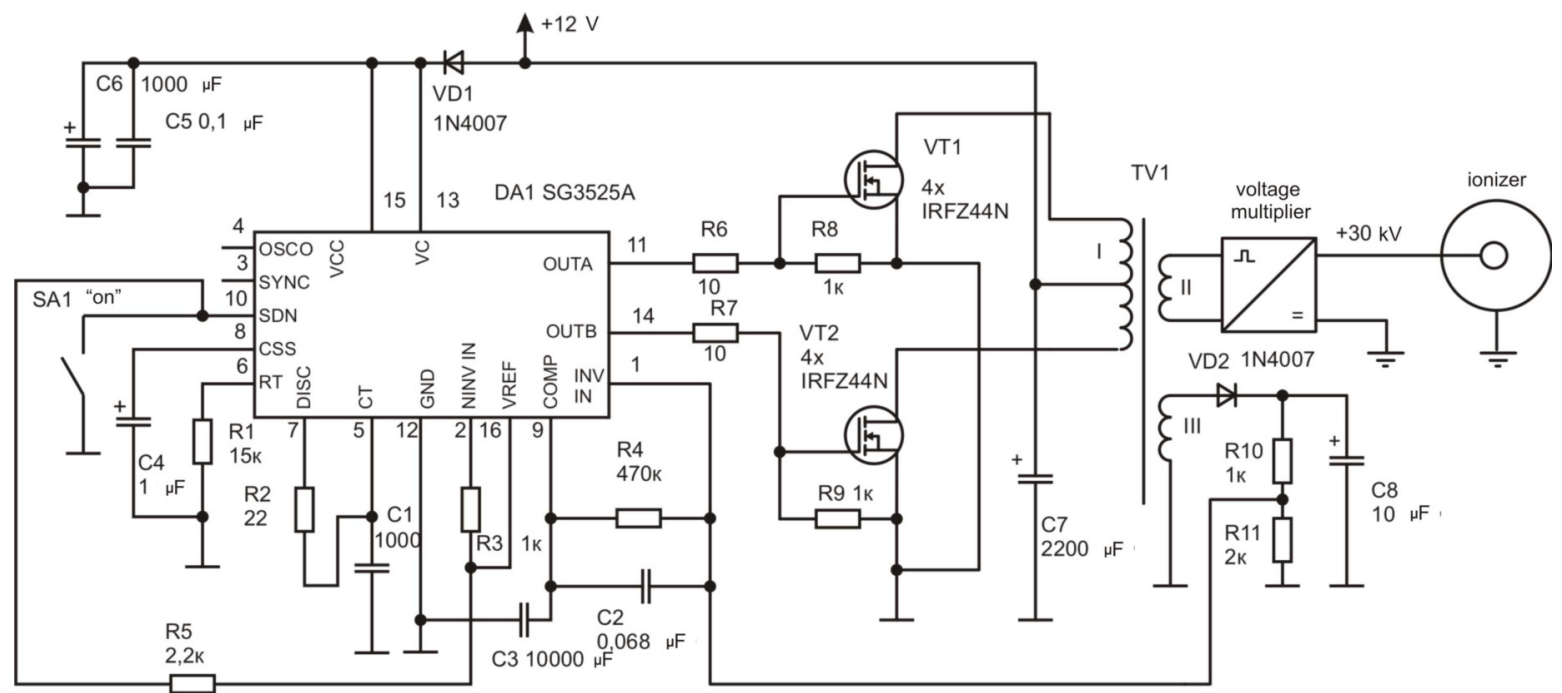

Fig. 3. Electrical schematic circuit of an inverter high voltage source

Assignment of elements and circuit operation (Fig. 3). The capacitor C1, resistor R2 - these are elements on the values of which the operating frequency of the controller depends. $\mathrm{C} 4$ - the soft start time of the circuit depends on the value of this capacitor. The duration of the dead time interval also depends on the value of the resistor R2. Outputs 1 and 2 of the DA1 chip are the inputs of the error amplifier. Since this control module is designed to operate as part of a powerful converter, a soft start circuit is assembled on this converter. That is, when the circuit is turned on, at the first moment of time, the output pulses duration of powerful key control is minimal. As the capacitor $\mathrm{C} 2$ charges, their duration increases to the desired value. The capacitors C3, C5 and C6 are filters. The feedback voltage from the winding of the transformer TV1 after rectification by the diode VD2 and filtering is fed to input 1 (control and error check). The diode VD1 ensures the safety of PWM (pulse-width modulation) when turned on the supply voltage in the reverse polarity.

Transistors VT1 and VT2 form a voltage inverter. Each of them is composed of four parallelconnected transistors of the same type IRFZ44 series. Due to the low spread of the parameters of individual transistors, pair selection is not required.

Source data and requirements for the multiplier:

- $U_{a}=3000 \mathrm{~V}$ (supply voltage amplitude);

- $F=50000 \mathrm{~Hz}, T=0.02 \mathrm{~ms}$ (frequency, period of supply voltage multiplier);

- $U_{\text {out }}=30000 \mathrm{~V}$ (output voltage);

- $\varepsilon_{\text {out }}=2 \%$ (maximum allowable drop in output voltage at full load);

- $U \sim=150 \mathrm{~V}$ (maximum permissible output pulsation amplitude);

- $I=40 \mathrm{~mA}$ (maximum load current);

- $m$ - even (there is a special requirement - the multiplication factor must be even).

Designing an inverter high-voltage source includes: determining operating modes and selecting power transistors; calculation and selection of the voltage multiplier elements; determination of design parameters of a power transformer.

\section{Results and discussion}

Volumetric mounting is considered classic for multipliers either on mounting racks or directly by the outputs of parts. If the installation is subsequently filled with a compound, the block is monolithic and non-repairable. The second option (repairable) involves the location of parts on a printed-circuit board for one-sided or two-sided installation. Subsequently, the board is covered with a special varnish.

The most technologically advanced option is the use of surface mount devices (SMD). In this case, it is necessary to design the board so that it is small in size, fully SMD, easily scalable. 
As a result of the design, a board with a size of $35 \times 45 \mathrm{~mm}$ is obtained, with mounts in the corners under a standard rack. The multiplier is scalable; the required number of modules is connected in series.

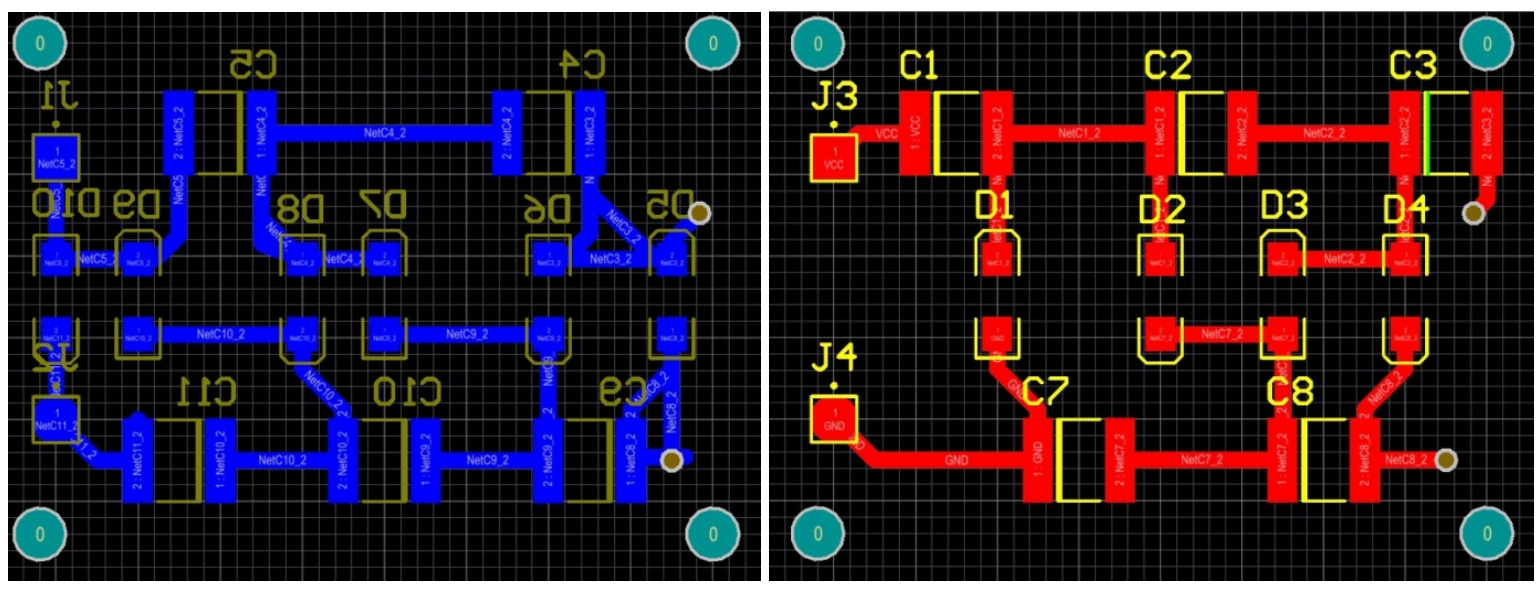

Fig. 4. Printed-circuit board for surface mounting

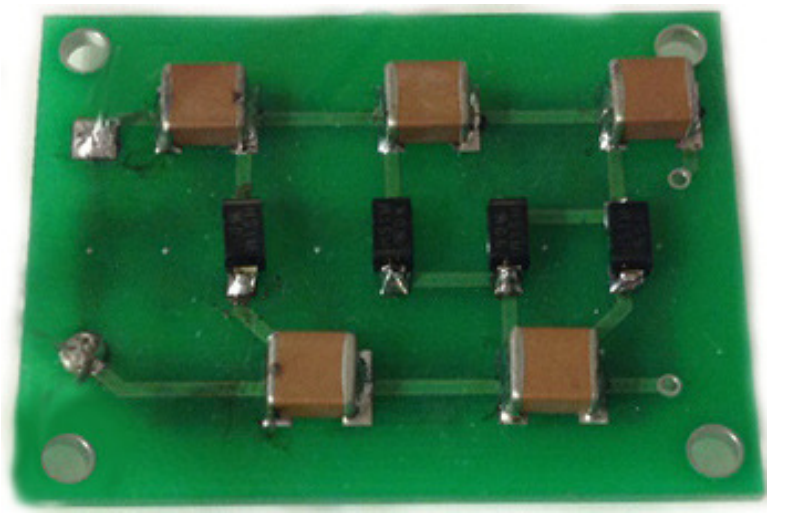

Fig. 5. Appearance of the printed-circuit board multiplier on SMD components

The operation of the voltage multiplier is under load. Let the duration of the passive phase, i.e. the time interval when all the diodes are off-state - from the beginning of a half-cycle to the moment when one of the multiplier diodes is on-state, is equal to $\theta$. The time during which one diode is onstate is denoted by $\theta 1$. As shown in Fig. 6., the diodes in the active phase of a half-cycle go into onstate one at a time, starting from the end of the circuit and further towards the beginning (it is clear that only odd or only even diodes will be on-state depending on the half-cycle). In fact, the time interval during which various on-state diodes are different, but these differences are much shorter than the half-cycle duration, and in any case this approximation will be more accurate than used earlier, when it was assumed that $\theta 1=0$.

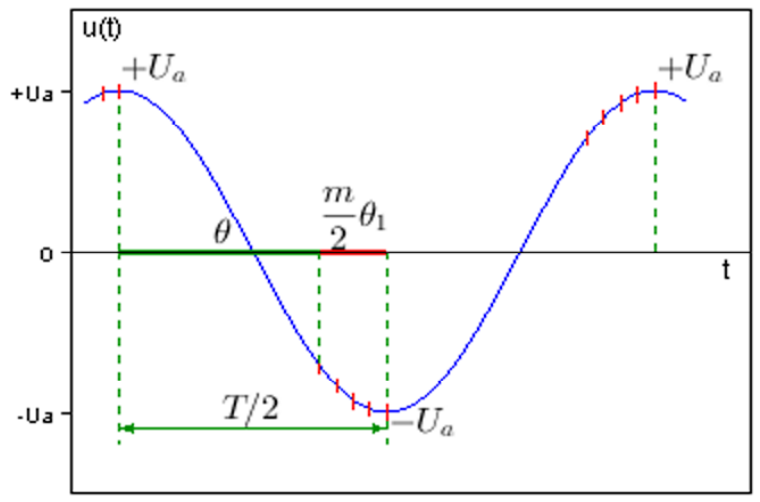

Fig. 6. Supply voltage graph 
In Fig. 7 plots of the passive phase duration of a half-cycle versus the load current for a given multiplier $\left(m=8, U_{a}=1000 \mathrm{~V}, F=100 \mathrm{kHz}, C=0.01 \mu \mathrm{F}\right)$ are presented when the load current changes from 0 to $3 \mathrm{~mA}$. The upper limit of the current change corresponds to a voltage drop under load of about $30 \%$ of the rated output voltage, thus, the current range under consideration covers almost all significant multiplier operating modes (it is impractical to use the multiplier for voltage drops greater than these values).

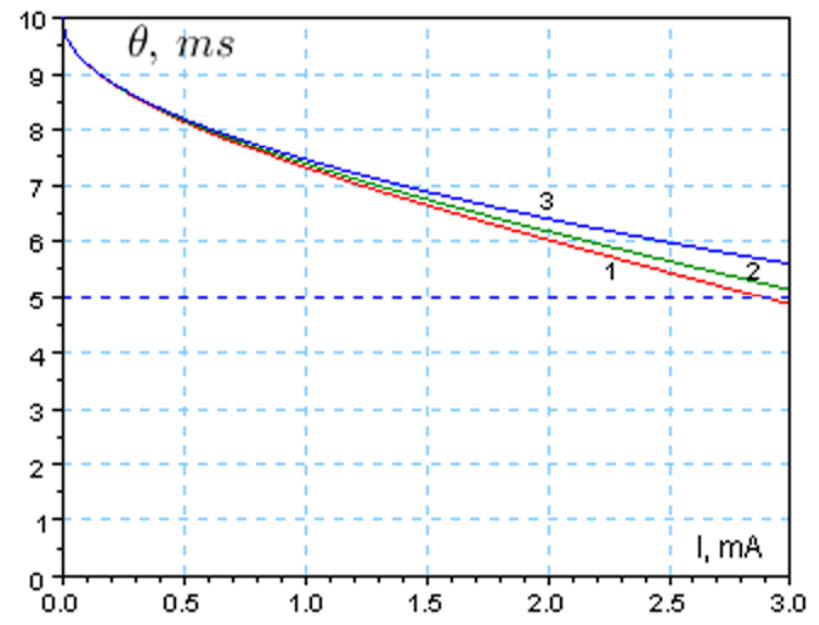

Fig. 7. Dependences of the passive half-cycle phase duration on the load current

Line 1 (red) is plotted according to the most accurate values. Line 2 (green) corresponds to the approximate calculation. Line 3 (blue) is constructed using an approximate formula, which is inferior in accuracy to line 2. In Fig. 7 the value of $10 \mathrm{~ms}$ along the $\theta$ axis corresponds to half of the period $(T / 2)$, and the value of $5 \mathrm{~ms}$ (the level is indicated by a blue dotted line in the graph) corresponds to a quarter of the period (T/4). As we expected, at zero current, $\theta$ turns into T/2. And at high load currents, the duration of the passive phase becomes even less than a quarter of the period, which means that the switching of the diodes begins even before the value $u(t)$ crosses the zero level.

Let us plot the dependence of this value on the load current (Fig. 8).

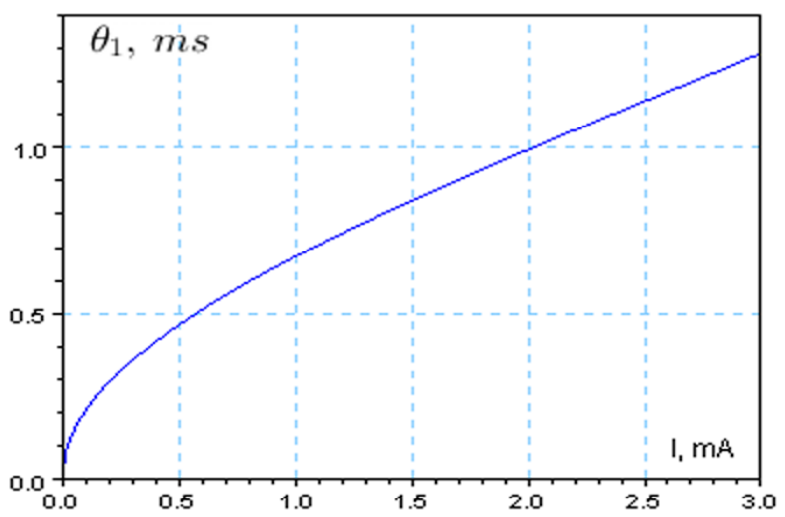

Fig. 8. Dependence of the on-state diode time on the load current

The graph shows that this value, as expected, is zero in an ideal multiplier in the absence of load. In the initial section of the dependence, one can observe a very high growth rate of the value, i.e. even at low load currents, the effect of switching time on the circuit operation becomes noticeable.

\section{Conclusions}

The development of a high voltage device for spraying systems should be based on specific operating conditions and parameters. It is preferable to use a multiplier circuit board on SMD components. It allows to arrange the required number of modules to create the optimal voltage on the electrodes of the ionizer. The presented analysis of the multiplier operation indicates that, when the load is connected, a voltage drop of up to $30 \%$ occurs, when the load current changes from 0 to $3 \mathrm{~mA}$. 


\section{References}

[1] Matthews G.A. Pesticide Application Methods. 3rd ed. - Wiley-Blackwell Science Ltd., 2000. $448 \mathrm{p}$.

[2] Antonov S.N., Gabriyelyan S.Z., Mastepanenko M.A., Zorina E.B., Nozdrovicky L The device of magnetic processing of water for boiler greenhouse. Research in agricultural engineering. Vol. 62, 2016, Special Issue: pp. 27-33.

[3] Архипов В. А. Аэрозольные системы и их влияние на жизнедеятельность (Aerosol systems and their influence on vital functions) / V.A. Arkhipov, U. M. Sheremetyeva. - Tomsk: TPU Publishing House, 2007. - 136 pp. (In Russian).

[4] Avdeeva, V.N., Antonov S.N., Molchanov A.G., Devederkin I.V. Disinfecting of winter wheatgrain withelectrophysical factors. Engineering for rural development, Jelgava Latvia, 2017, pp. 323-327.

[5] Турчин И.А. Электризация рабочих жидкостей (Electrification of working fluids). // Journal of Plant Protection. - №9. - 1987. - P. 29-36. (In Russian).

[6] Nasr G.G., Yule A.J., Bendig L. Industrial Sprays and Atomization: Design, Analysis and Applications. Springer-Verlag London, 2002. $501 \mathrm{p}$.

[7] Antonov S., Nikitenko G., Adoshev A., Dorozhko S., Devederkin I., Method of effective spraying plants with drop charge of fertilizer solution by ionized air. Engineering for rural development, Jelgava Latvia, 2018, pp. 519-523.

[8] Antonov S., Development of a high voltage source for spraying plants with ionized aerosol. Engineering for rural development, Jelgava Latvia, 2019, pp. 808-813. 